\title{
Interpretations of quantum theory: A map of madness
}

\author{
Adán Cabello ${ }^{1}$ \\ Departamento de Física Aplicada II, Universidad de Sevilla, E-41012 Sevilla, \\ Spain.
}

\begin{abstract}
Motivated by some recent news ${ }^{1}$, a journalist asks a group of physicists: "What's the meaning of the violation of Bell's inequality?" One physicist answers: "It means that non-locality is an established fact". Another says: "There is no non-locality; the message is that measurement outcomes are irreducibly random". A third one says: "It cannot be answered simply on purely physical grounds, the answer requires an act of metaphysical judgement" a . Puzzled by the answers, the journalist keeps asking questions about quantum theory: "What is teleported in quantum teleportation?" "How does a quantum computer really work?" Shockingly, for each of these questions, the journalist obtains a variety of answers which, in many cases, are mutually exclusive. At the end of the day, the journalist asks: "How do you plan to make progress if, after 90 years of quantum theory, you still don't know what it means? How can you possibly identify the physical principles of quantum theory or expand quantum theory into gravity if you don't agree on what quantum theory is about?" Here we argue that it is becoming urgent to solve this too long lasting problem. For that, we point out that the interpretations of quantum theory are, essentially, of two types and that these two types are so radically different that there must be experiments that, when analyzed outside the framework of quantum theory, lead to different empirically testable predictions. Arguably, even if these experiments do not end the discussion, they will add new elements to the list of strange properties that some interpretations must have, therefore they will indirectly support those interpretations that do not need to have all these strange properties.
\end{abstract}

As Mermin points out, "quantum theory is the most useful and powerful theory physicists have ever devised. Yet today, nearly 90 years after its formulation, disagreement about the meaning of the theory is stronger than ever. New interpretations appear every day. None ever disappear" 5 . This situation is odd and is arguably an obstacle for scientific progress, or at least for a certain kind of scientific progress. The periodic efforts of listing and comparing the increasing number of interpretations ${ }^{6-9}$ show that there is something persistent since the formulation of quantum theory: Interpretations are essentially of two types; those that view quantum probabilities of measurement outcomes as determined by intrinsic properties of the world and those that do not. Here we call them "type-I" and "type-II", respectively. In Table I some interpretations are classified according to this criterion and some extra details are given.

Two observations can be made in the light of Table I.

Observation one: The extended belief that both types of interpretations "yield the same empirical consequences" and therefore "the choice between them cannot be made simply on purely physical grounds but it requires an act of metaphysical judgement" 4 is arguably wrong. The two types are so different

\footnotetext{
a) All of our characters are fictitious. However, it might be interesting to compare their points of view with those in Refs. ${ }^{2-4}$, respectively
}

that is very unlikely that distinguishing between them is forever out of reach of scientific method. In fact, by making some reasonable assumptions it can be shown ${ }^{39}$ that type-I interpretations must have more strange properties than those suggested by previous approaches ${ }^{40-42}$.

Observation two: The proposed principles for reconstructing quantum theory ${ }^{43-50}$ are neutral with respect to interpretations. This may be a drawback. Although these approaches can lead to the mathematical structure of the theory, the need to remain neutral may be an obstacle for identifying physical principles. This becomes evident when the proposed principles are examined in the light of some specific interpretations (e.g., QBism ${ }^{33-35}$ ). Then, not all of them are equally important: Some simply follow from the assumed interpretational framework; only a few of them give some physical insight. This suggests that there may be a bonus in nonneutral reconstructions of quantum theory. This will be developed elsewhere.

This Table and these observations should be taken as motivations for further work. The fact that they may be controversial by themselves, and the interest showed by many colleagues, justify presenting them separately from any of these works.

Acknowledgements: This work was supported by the FQXi large grant project "The Nature of Information in Sequential Quantum Measurements" 


\begin{tabular}{|c|c|c|c|c|c|}
\hline & & $\psi$-Epistemic & & About knowledge & \begin{tabular}{|l|} 
About belief \\
\end{tabular} \\
\hline $\begin{array}{c}\text { Type-I } \\
\text { (intrinsic realism) }\end{array}$ & \begin{tabular}{|l|}
\multicolumn{1}{|c|}{$\psi$-Untic } \\
Bohmian mechanics ${ }^{10,11}$ \\
Many worlds $^{12,13}$ \\
Modal $^{14,15}$ \\
Bell's "beables"16 $^{*}$ Collapse theories*17,18 $^{2}$ \\
\end{tabular} & \begin{tabular}{|l|}
\multicolumn{1}{|c|}{$\psi$-Epistemic } \\
Einstein $^{19}$ \\
Ballentine $^{20}$ \\
Consistent histories $^{21,22}$ \\
Spekkens $^{23}$
\end{tabular} & $\begin{array}{c}\text { Type-II } \\
\text { (participatory realism) }\end{array}$ & $\begin{array}{l}\text { Copenhagen }^{24,25} \\
\text { Wheeler }^{26,27} \\
\text { Relational }^{28,29} \\
\text { Zeilinger }^{3,30} \\
\text { No "interpretation" } 31 \\
\text { Brukner }\end{array}$ & QBism $^{33-35}$ \\
\hline
\end{tabular}

TABLE I. Some interpretations of quantum theory classified according to whether they view probabilities of measurement outcomes as determined or not by intrinsic properties of the observed system. Type-I interpretations are defined as those in which the probabilities of measurement outcomes are determined by intrinsic properties of the observed system. Type-I interpretations can be " $\psi$-ontic" ${ }^{36}$, if they view the quantum state as an intrinsic property of the observed system, or " $\psi$-epistemic" ${ }^{36}$, if they view the quantum state as representing knowledge of an underlying objective reality in a sense somewhat analogous to that in which a state in classical statistical mechanics assigns a probability distribution to points in phase space. "Type-II interpretations" are defined as those which do not view the probabilities of measurement outcomes of quantum theory as determined by intrinsic properties of the observed system. Type-II interpretations do not deny the existence of an objective world but, according to them, quantum theory does not deal directly with intrinsic properties of the observed system, but with the experiences an observer or agent has of the observed system. Type-II interpretations can be "about knowledge" if they view the quantum state as an observer's knowledge about the results of future experiments, or "about belief" if they view the quantum state as an agent's expectations about the results of future actions. This table is based on a similar table made by Leifer ${ }^{37}$, but has many significant differences and incorporates suggestions from many colleagues (see acknowledgements). The term "participatory realism" (inspired by Wheeler's "participatory universe" ${ }^{27}$ ) was suggested by $\mathrm{Fuchs}^{38}$. *: Collapse theories modify or supplement the unitary formalism of quantum theory; therefore, they are not pure interpretations.

and project FIS2014-60843-P (MINECO, Spain) with FEDER funds. I thank D. Z. Albert, M. Araújo, L. E. Ballentine, H. R. Brown, Č. Brukner, J. Bub, G. Chiribella, D. Dieks, C. A. Fuchs, R. B. Griffiths, M. Kleinmann, J.-A. Larsson, M. Leifer, O. Lombardi, N. D. Mermin, M. P. Müller, R. Schack, C. Timpson, L. Vaidman, D. Wallace, A. G. White, and A. Zeilinger for conversations and suggestions for improving the table.

${ }^{1}$ Hensen, B. et al., Loophole-free Bell inequality violation using electron spins separated by 1.3 kilometres, Nature 526, 682-686 (2015).

${ }^{2}$ Gisin, N. Non-realism: Deep thought or a soft option? Found. Phys. 42, 80-85 (2012).

${ }^{3}$ Zeilinger, A. The message of the quantum. Nature 438, 743 (2005).

${ }^{4}$ Polkinghorne, J. Physics and theology. Europhysics News 45, No. 1, 28-31 (2014).

${ }^{5}$ Mermin, N. D. Quantum mechanics: Fixing the shifty split. Phys. Today 65, No. 7, 8-10 (2012).

${ }^{6}$ Belinfante, F. J. A Survey of Hidden-Variables Theories (Pergamon Press, New York, 1973).

${ }^{7}$ Jammer, M. The Philosophy of Quantum Mechanics: The Interpretations of Quantum Mechanics in Historical Perspective (Wiley, New York, 1974).

${ }^{8}$ Bub, J. Interpreting the Quantum World (Cambridge University Press, Cambridge, 1997).

${ }^{9}$ Dickson, W. M. Quantum Chance and Nonlocality: Probability and Nonlocality in the Interpretation of Quantum Mechanics (Cambridge University Press, Cambridge, 1998).

${ }^{10}$ Bohm, D. A suggested interpretation of the quantum theory in terms of hidden variables. I \& II. Phys. Rev. 85, 166-193 (1952).

${ }^{11}$ Goldstein, S. Bohmian mechanics. In The Stanford Encyclopedia of Philosophy, edited by E. N. Zalta (2013).

${ }^{12}$ Everett III, H. "Relative state" formulation of quantum mechanics. Rev. Mod. Phys. 29, 454-462 (1957).

${ }^{13}$ Vaidman, L. Many-worlds interpretation of quantum mechanics.
In The Stanford Encyclopedia of Philosophy, edited by E. N. Zalta (2015).

${ }^{14}$ Van Fraassen, B. C., A formal approach to the philosophy of science. In Paradigms and Paradoxes: The Philosophical Challenge of the Quantum Domain, edited by R. Colodny (University of Pittsburgh Press, Pittsburgh), pp. 303-366 (1972).

${ }^{15}$ Lombardi, O. \& Dieks, D. Modal interpretations of quantum mechanics. In The Stanford Encyclopedia of Philosophy, edited by E. N. Zalta (2012).

${ }^{16}$ Bell, J. S. The theory of local beables, Epistemological Lett. No. 9 (March 1976).

${ }^{17}$ Ghirardi, G. C., Rimini, A. \& Weber, T. Unified dynamics for microscopic and macroscopic systems. Phys. Rev. D 34, 470-491 (1986).

${ }^{18}$ Ghirardi, G. C., Collapse theories. In The Stanford Encyclopedia of Philosophy, edited by E. N. Zalta (2011).

${ }^{19}$ Einstein, A. Physics and reality. J. Franklin Inst. 221, 349-382 (1936).

${ }^{20}$ Ballentine, L. E. The statistical interpretation of quantum mechanics. Rev. Mod. Phys. 42, 358-381 (1970).

${ }^{21}$ Griffiths, R. B. Consistent histories and the interpretation of quantum mechanics. J. Stat. Phys. 36, 219-272 (1984).

${ }^{22}$ Griffiths, R. B. The consistent histories approach to quantum mechanics. In The Stanford Encyclopedia of Philosophy, edited by E. N. Zalta (2014).

${ }^{23}$ Spekkens, R. W. Evidence for the epistemic view of quantum states: A toy theory. Phys. Rev. A 75, 032110 (2007).

${ }^{24} \mathrm{Bohr}$, N. The Philosophical Writings of Niels Bohr, edited by J. Faye \& H. J. Folse (Ox Bow Press, Woodbridge, Connecticut, 1998).

${ }^{25}$ Faye, J. Copenhagen interpretation of quantum mechanics. In The Stanford Encyclopedia of Philosophy, edited by E. N. Zalta (2014).

${ }^{26}$ Wheeler, J. A. Law without law. In Quantum Theory and Measurement, edited by J. A. Wheeler \& W. H. Zurek (Princeton University Press, Princeton, New Jersey, 1983), pp. 182-213.

${ }^{27}$ Wheeler, J. A. At Home in the Universe (American Institute of Physics Press, Woodbury, NY, 1994).

${ }^{28}$ Kochen, S. A new interpretation of quantum mechanics. In Symposium on the Foundations of Modern Physics: 50 Years of the 
Einstein-Podolsky-Rosen Experiment, edited by P. J. Lahti \& P. Mittelstaedt (World Scientific, Singapore, 1985), pp. 151-169.

${ }^{29}$ Rovelli, C. Relational quantum mechanics. Int. J. Th. Phys. 35, 1637-1678 (1996).

${ }^{30}$ Zeilinger, A. A foundational principle for quantum mechanics. Found. Phys. 29, 631-643 (1999).

${ }^{31}$ Fuchs, C. A. \& Peres, A. Quantum theory needs no 'interpretation'. Phys. Today 53, No. 3, 70-71 (2000).

${ }^{32}$ Brukner, C. . On the quantum measurement problem. To appear in the Proceedings of the Conference "Quantum UnSpeakables II: 50 Years of Bell's Theorem" (Vienna, 19-22 June 2014); arXiv:1507.05255.

${ }^{33}$ Fuchs, C. A. QBism, the perimeter of quantum Bayesianism. arXiv: 1003.5209 .

${ }^{34}$ Fuchs, C. A. \& Schack, R. Quantum-Bayesian coherence. Rev. Mod. Phys. 85, 1693-1714 (2013).

${ }^{35}$ Fuchs, C. A., Mermin, N. D. \& Schack, R. An introduction to QBism with an application to the locality of quantum mechanics. Am. J. Phys. 82, No. 8, 749-754 (2014).

${ }^{36}$ Harrigan, N. \& Spekkens, R. W. Einstein, incompleteness, and the epistemic view of quantum states. Found. Phys. 40, 125-157 (2010).

${ }^{37}$ Leifer, M. S. Is the wavefunction real? Talk at the 12 th Biennial IQSA Meeting Quantum Structures (Olomouc, Czech Republic, 2014).

${ }^{38}$ Fuchs, C. A. On participatory realism. arXiv:1601.04360.

${ }^{39}$ Cabello, A., Gu, M., Gühne, O., Larsson, J.-Å. \& Wiesner, K.
Thermodynamical cost of some interpretations of quantum theory. Phys. Rev. A 94, 052127 (2016).

${ }^{40}$ Kochen, S. \& Specker, E. P. The problem of hidden variables in quantum mechanics. J. Math. Mech. 17, 59-87 (1967).

${ }^{41}$ Bell, J. S. On the Einstein Podolsky Rosen paradox. Physics 1, 195-200 (1964).

${ }^{42}$ Pusey, M. F., Barrett, J. \& Rudolph, T. On the reality of the quantum state. Nature Physics 8, 475-478 (2012).

${ }^{43}$ Hardy, L. Quantum theory from five reasonable axioms. quant$\mathrm{ph} / 0101012$.

${ }^{44}$ Hardy, L. Reformulating and reconstructing quantum theory. arXiv: 1104.2066

${ }^{45}$ Dakić, B. \& Brukner, Č. Quantum theory and beyond: Is entanglement special? In Deep Beauty. Understanding the Quantum World through Mathematical Innovation, edited by H. Halvorson (Cambridge University Press, New York, 2011), pp. 365-392.

${ }^{46}$ Masanes, L. \& Müller, M. P. A derivation of quantum theory from physical requirements. New J. Phys. 13, 063001 (2011).

${ }^{47}$ Chiribella, G., D'Ariano, G. M. \& Perinotti, P. Informational derivation of quantum theory. Phys. Rev. A 84, 012311 (2011).

${ }^{48}$ Cabello, A. Simple explanation of the quantum violation of a fundamental inequality. Phys. Rev. Lett. 110, 060402 (2013).

${ }^{49}$ Barnum, H., Müller, M. P. \& Ududec, C. Higher-order interference and single-system postulates characterizing quantum theory. New J. Phys. 16, 123029 (2014).

${ }^{50}$ Chiribella, G. \& Yuan, X. Measurement sharpness cuts nonlocality and contextuality in every physical theory, arXiv:1404.3348. 